\title{
Behavioral deficits in an Angelman syndrome model: Effects of genetic background and age
}

\author{
Hsien-Sung Huang ${ }^{\mathrm{a}}$, Andrew J. Burns ${ }^{\mathrm{a}}$, Randal J. Nonneman ${ }^{\mathrm{b}}$, Lorinda K. Baker ${ }^{\mathrm{b}, \mathrm{c}}$, Natallia \\ V. Riddick ${ }^{\mathrm{b}, \mathrm{c}}$, Viktoriya D. Nikolova ${ }^{\mathrm{c}}$, Thorfinn T. Riday ${ }^{\mathrm{a}}$, Koji Yashiro ${ }^{\mathrm{a}}$, Benjamin D.

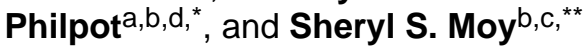 \\ a Department of Cell Biology and Physiology, University of North Carolina School of Medicine, \\ Chapel Hill, NC 27599, USA \\ ${ }^{b}$ Carolina Institute for Developmental Disabilities, University of North Carolina School of \\ Medicine, Chapel Hill, NC 27599, USA \\ c Department of Psychiatry, University of North Carolina School of Medicine, Chapel Hill, NC \\ 27599, USA \\ d UNC Neuroscience Center, University of North Carolina School of Medicine, Chapel Hill, NC \\ 27599, USA
}

\begin{abstract}
Angelman syndrome (AS) is a severe neurodevelopmental disorder associated with disruption of maternally inherited $U B E 3 A$ (ubiquitin protein ligase $\mathrm{E} 3 \mathrm{~A}$ ) expression. At the present time, there is no effective treatment for AS. Mouse lines with loss of maternal $U b e 3 a\left(U_{b e} 3 a^{m-/ p+}\right)$ recapitulate multiple aspects of the clinical AS profile, including impaired motor coordination, learning deficits, and seizures. Thus, these genetic mouse models could serve as behavioral screens for preclinical efficacy testing, a critical component of drug discovery for AS intervention. However, the severity and consistency of abnormal phenotypes reported in $U b e 3 a^{m-/ p+}$ mice can vary, dependent upon age and background strain, which is problematic for the detection of beneficial drug effects. As part of an ongoing AS drug discovery initiative, we characterized Ube $3 a^{m-/ p+}$ mice on either a 129S7/SvEvBrd-Hprt $t^{b-m 2}$ (129) or C57BL/6J (B6) background across a range of functional domains and ages to identify reproducible and sufficiently large phenotypes suitable for screening therapeutic compounds. The results from the study showed that Ube $3 a^{m-/ p+}$ mice have significant deficits in acquisition and reversal learning in the Morris water maze. The findings also demonstrated that $U b e 3 a^{m-/ p+}$ mice exhibit motor impairment in a rotarod task, hypoactivity, reduced rearing and marble-burying, and deficient fear conditioning. Overall, these profiles of abnormal phenotypes can provide behavioral targets for evaluating effects of novel therapeutic strategies relevant to AS.
\end{abstract}

\section{Keywords}

Angelman syndrome; Conditioned fear; Inbred strain; Morris water maze; Reversal learning; UBE3A

\footnotetext{
(C) 2013 Elsevier B.V. All rights reserved.

* Corresponding author at: University of North Carolina, CB\# 7545, 115 Mason Farm Road, University of North Carolina School of Medicine, Chapel Hill, NC 27599-7545, USA. Tel.: +1 919966 0025; fax: +1 919966 3870. bphilpot@ med.unc.edu (B.D. Philpot). ** Corresponding author at: Carolina Institute for Developmental Disabilities, CB\# 7146, University of North Carolina School of Medicine, Chapel Hill, NC 27599, USA. Tel.: +1 919966 3082; fax: +1 919966 5657. ssmoy@ med.unc.edu (S.S. Moy)..
} 


\section{Introduction}

Angelman syndrome (AS) is a severe genomic imprinting disorder with phenotypes that typically manifest early in childhood. AS is characterized by intellectual disability, speech impairment, motor dysfunction, sleep disturbances, epilepsy, inappropriate laughter, and an unusually happy demeanor [1,2]. In most cases, AS arises from the deletion or mutation of maternal $U B E 3 A[3,4]$, which encodes ubiquitin protein ligase E3A. In neurons, only the maternal copy of $U B E 3 A$ is active, while the paternally inherited $U B E 3 A$ allele is silenced [5,6]. Evidence from mouse lines with targeted disruption of the maternal $U b e 3 a$ allele supports an important role for UBE3A in neuronal morphology, synaptic function, and the maturation of neocortical circuits in the brain [7-10]. Maternal Ube3a-deficient mice (Ube $3 a^{m-/ p+}$ mice) have abnormal phenotypes that resemble many of the clinical symptoms observed in AS, including motor dysfunction, cognitive deficits, and enhanced susceptibility to seizures [11]. Ube $3 a^{m-/ p+}$ mice also have overt deficiencies in hippocampal long-term potentiation and dendritic spine density, in line with deficits in behavioral tasks, such as contextual fear conditioning and spatial learning in the Morris water maze, which are mediated by the hippocampus $[9,11-14]$.

Although no effective treatment currently exists for AS, recent findings utilizing genetic mouse models for the disorder have suggested that abnormal phenotypes can be rescued $[12,14,15]$. For example, van Woerden et al. [14] were able to genetically rescue the deficits in rotarod performance, quadrant selectivity in the water maze, and contextual fear conditioning that characterize $U b e 3 a^{m-/ p+}$ mice. Our research group recently demonstrated that topoisomerase inhibitors can unsilence paternally inherited Ube3a [16], but the possible beneficial effects of these drugs on abnormal behaviors relevant to AS have not yet been established. While the $U b e 3 a^{m-/ p+}$ mice provide a well-validated model for preclinical efficacy testing, there is a critical need to identify the optimal phenotypes to target for reversal in drug discovery studies. Importantly, there are known strain-specific differences in behavioral phenotypes [11]. Moreover, even in the most carefully controlled studies, it can be difficult to find behavioral phenotypes sufficiently penetrant for inter-species, interlaboratory, and intra-laboratory reproducibility [17]. We therefore sought to identify AS phenotypes of sufficient magnitude and consistency to be suitable for screening potential therapeutics. Toward this goal, the present studies evaluated $U b e 3 a^{m-/ p+}$ mice on two different genetic backgrounds, either 129S7/SvEvBrd-Hprt ${ }^{b-m 2}$ (129) or C57BL/6J (B6), using multi-component phenotyping regimens and testing at different ages. Because clinical studies have linked genotype to differential developmental trajectories in AS [18], separate cohorts of B6 mice were evaluated, beginning from either adolescence or adulthood, to examine both phenotypic trajectories and reproducibility.

\section{Materials and methods}

\subsection{Animals}

Subjects were heterozygous mice with maternal deficiency of $U b e 3 a\left(U b e 3 a^{m-/ p+}\right)$ and wild type $\left(U b e 3 a^{m+/ p+}\right)$ littermates, on two different background strains: 129S7/SvEvBrd$\mathrm{Hprt}^{b-m 2}$ (129) and C57BL/6J (B6) [11]. The $U b e 3 a^{m+/ p-}$ mice on a 129 strain background were developed by the Beaudet laboratory [11] and were obtained from Jackson Laboratory (Bar Harbor, ME). The Ube $3 a^{m-/ p+}$ mice on a B6 background were originally developed by the Beaudet laboratory [11] and were backcrossed at least 10 generations onto the B6 strain by Dr. Yong-hui Jiang. All mice in the B6 groups for the present study were offspring from breeding pairs obtained from Dr. Yong-hui Jiang. One group of 129-background mice and four separate cohorts of B6-background mice were tested for behavior (described below). Mice were group-housed in ventilated cages, with free access to water and Prolab RMH 3000 chow. The housing room had a 12-h light/dark cycle (lights off at 7:00 p.m.). 
Genotyping was conducted by PCR from tail tissue samples. All procedures were conducted in strict compliance with the policies on animal welfare of the National Institutes of Health and the University of North Carolina (stated in the "Guide for the Care and Use of Laboratory Animals," Institute of Laboratory Animal Resources, National Research Council, 1996 edition).

\subsection{Test groups}

129-background mice-Subjects were $10 \mathrm{Ube} 3 \mathrm{a}^{\mathrm{m+} / \mathrm{pt}}$ mice (5 males and 5 females) and $11 \mathrm{Ube} 3 \mathrm{a}^{m-/ p+}$ mice (4 males and 7 females), derived from 7 litters. Testing began when mice were between 7 and 8 weeks in age.

B6-background mice (cohort 1)—Subjects were $14 \mathrm{Ube}^{3 \mathrm{a}^{m+/ p+}}$ mice (8 male and 6 female) and $11 \mathrm{Ube} 3 \mathrm{a}^{m-/ p+}$ mice (4 male and 7 female), derived from 5 litters. Testing began during the adolescent period, when mice were $4-5$ weeks in age.

B6-background mice (cohort 2)-Subjects were $19 \mathrm{Ube}^{3} \mathrm{a}^{\mathrm{m+} / \mathrm{p}+}$ and $16 \mathrm{Ube} 3 \mathrm{a}^{\mathrm{m}-\mathrm{p}^{+}}$ mice, all male, derived from 9 litters. Testing began in adulthood, when mice were 12-13 weeks in age.

B6-background mice (cohort 3)—One set of male subjects (10 Ube $3 a^{m+/ p+}$ and 7 $U b e 3 a^{m-/ p+}$ mice, derived from 6 litters) was used to confirm results from the marbleburying assay. Mice were given two tests, one at age 18-20 weeks, and a second test at age 19-21 weeks.

B6-background mice (cohort 4)-Subjects in the conditioned fear group were 13 $U b e 3 a^{m+/ p+}$ and $12 U b e 3 a^{m-/ p+}$ mice, all male, derived from 6 litters. Testing began when mice were between 11 and 13 weeks of age.

129- and B6-background mice for body weight evaluation-Data on body weight were compiled for offspring from the same breeding colony that provided mice for the behavioral studies, with one weight measure per mouse. Subjects on the 129-background were $157 \mathrm{Ube} 3 \mathrm{a}^{m+/ p+}$ mice ( 82 males and 75 females) and $101 \mathrm{Ube} 3 \mathrm{a}^{m-/ p+}$ mice (53 males and 48 females). Subjects on the B6-background were $337 \mathrm{Ube} 3 a^{m+/ p+}$ mice (159 males and 178 females) and $244 U b e 3 a^{m-/ p+}$ mice (139 males and 105 females).

To blind experimenters to genotype, all mice for behavioral testing were given new identification codes, and all genotype information was removed from cage cards. Mice in the first three groups, the 129 mice and first two cohorts of B6 mice, were evaluated for activity (1-h duration), grip strength in a wire-hang test, motor coordination on an accelerating rotarod, sensorimotor gating in an acoustic startle test, and acquisition/reversal learning in the Morris water maze. Only a subset of the second cohort of B6 mice was tested in the water maze (10 Ube $3 a^{m+/ p+}$ and $11 \mathrm{Ube} 3 a^{m-/ p+}$ ). Following the initial battery of tests, the first two B6-background cohorts were given further activity, grip strength, rotarod, and acoustic startle tests, in order to evaluate changes in behavior across time (see Table 1 for age at each test).

The 129 and first cohort of B6 mice were also assessed for sociability in a 3-chamber choice task. The second cohort of B6 mice was assessed for digging behavior in a marble-burying assay as an index of repetitive responses. 


\subsection{Testing procedures}

2.3.1. Activity-Exploratory activity in a novel environment was assessed by 1-h trials in a photocell-equipped automated chamber $(41 \mathrm{~cm} \times 41 \mathrm{~cm} \times 30 \mathrm{~cm}$; Versamax system, Accuscan Instruments). Measures were taken of total distance traveled, number of rearing movements, and time spent in the center of the field. Activity chambers were contained inside sound-attenuating boxes equipped with ceiling-mounted lights and fans.

2.3.2. Wire hang test for grip strength-Each mouse was placed on a large metal cage lid. The lid was gently shaken to induce the mouse to grip the metal grid. The cage top was then inverted, and latency for the mouse to fall from the lid was recorded. The maximum trial length was $60 \mathrm{~s}$.

2.3.3. Rotarod performance-Mice were assessed for balance and motor coordination on an accelerating rotarod (Ugo-Basile, Stoelting Co., Wood Dale, Il). Revolutions per minute (rpm) were set at an initial value of 3 , with a progressive increase to a maximum of $30 \mathrm{rpm}$ across $5 \mathrm{~min}$, the maximum trial length. Test sessions consisted of 2 or 3 trials, with $45 \mathrm{~s}$ between each trial. Latency to fall, or to rotate off the top of the turning barrel, was measured by the rotarod timer.

2.3.4. Acoustic startle procedure-The acoustic startle measure was based on the reflexive whole-body flinch, or startle response, following exposure to a sudden noise. Animals were tested with a San Diego Instruments SR-Lab system, using published methods [19]. Briefly, mice were placed in a small Plexiglas cylinder within a larger, soundattenuating chamber (San Diego Instruments). The cylinder was seated upon a piezoelectric transducer, which allowed vibrations to be quantified and displayed on a computer. The chamber included a ceiling light, fan, and a loudspeaker for the acoustic stimuli (bursts of white noise). Background sound levels (70 dB) and calibration of the acoustic stimuli were confirmed with a digital sound level meter (San Diego Instruments). Each test session consisted of 42 trials, presented following a 5-min habituation period. There were 7 different types of trials: the no-stimulus trials, trials with the acoustic startle stimulus ( $40 \mathrm{~ms} ; 120 \mathrm{~dB}$ ) alone, and trials in which a prepulse stimulus ( $20 \mathrm{~ms}$; either $74,78,82,86$, or $90 \mathrm{~dB}$ ) had onset $100 \mathrm{~ms}$ before the onset of the startle stimulus. The different trial types were presented in blocks of 7, in randomized order within each block, with an average intertrial interval of $15 \mathrm{~s}$ (range: 10-20 s). Measures were taken of the startle amplitude for each trial, defined as the peak response during a $65-\mathrm{ms}$ sampling window that began with the onset of the startle stimulus. Levels of PPI (prepulse inhibition) at each prepulse sound level were calculated as $100-[$ (response amplitude for prepulse stimulus and startle stimulus together/response amplitude for startle stimulus alone) $\times 100]$.

2.3.5. Sociability and preference for social novelty-Mice were tested in an automated 3-chambered box, using published methods [20,21]. Dividing walls had retractable doorways allowing access into each chamber. The automated box had photocells embedded in each doorway to allow quantification of entries and duration in each chamber of the social test box. The chambers of the apparatus were cleaned with water and dried with paper towels between each trial. At the end of each test day, the apparatus was sprayed with $70 \%$ ethanol and wiped clean with paper towels.

The choice test had two 10-min phases: (1) Habituation. The test mouse was first placed in the middle chamber and allowed to explore, with the doorways into the two side chambers open. (2) Sociability. After the habituation period, the test mouse was enclosed in the center compartment of the social test box, and an unfamiliar mouse (the stranger; a sex-matched C57BL/6J adult) was enclosed in a wire cage (Galaxy Cup, Spectrum Diversified Designs, 
Inc., Streetsboro, $\mathrm{OH}$ ) and placed in a side chamber. The location for the stranger alternated between the left and right sides of the social test box across subjects. An empty wire cage was placed in the opposite side to serve as a non-social novel object. Following placement of the stranger, the doors were re-opened, and the subject was allowed to explore the entire social test box. Measures were taken automatically of the amount of time spent in each chamber and the number of entries into each chamber. Measures of time spent sniffing each wire cage were recorded by a human observer blind to genotype.

2.3.6. Morris water maze-Mice were tested in the water maze, based on published methods [20,22]. The water maze consisted of a large circular pool (diameter $=122 \mathrm{~cm})$ partially filled with water ( $45 \mathrm{~cm}$ deep, $24-26{ }^{\circ} \mathrm{C}$ ), located in a room with numerous visual cues. Mice were tested for their ability to find an escape platform (diameter $=12 \mathrm{~cm}$ ) in 3 different learning phases: with a cued visible platform, acquisition in the hidden (submerged) platform test, and reversal learning with the hidden platform moved to the opposite quadrant. In each case, the criterion for learning was an average latency of $15 \mathrm{~s}$ or less to locate the platform across a block of 4 consecutive trials per day. In addition, at the end of the acquisition and reversal learning phases, mice were given 1-min probe trials with the platform removed. In these probe trials, spatial learning was measured by numbers of crosses over the location where the platform had been during the training trials, versus swimming over corresponding areas of the other three quadrants in the pool.

In the visible platform test, each animal was given 4 trials per day, across 3 days, to swim to an escape platform cued by a patterned cylinder extending above the surface of the water. For each trial, the mouse was placed in the pool at 1 of 4 possible locations (randomly ordered), and then given $60 \mathrm{~s}$ to find the cued platform. If the mouse found the platform, the trial ended, and the animal was allowed to remain $10 \mathrm{~s}$ on the platform before the next trial began. If the platform was not found, the mouse was placed on the platform for $10 \mathrm{~s}$, and then given the next trial. Measures were taken of latency to find the platform and swimming speed, via an automated tracking system (Ethovision, Noldus Information Technology, Wageningen, the Netherlands).

The following week, mice were evaluated for acquisition in the hidden platform test. Using the same procedure as described above, each animal was given 4 trials per day, for up to 9 days, to learn the location of the submerged platform. At the end of the day that the group met the 15-s criterion for learning, or else on day 9 of testing, mice were given a 1-min probe trial in the pool with the platform removed. Selective quadrant search was evaluated by measuring platform location crossings. In the week following the acquisition phase, mice were tested for reversal learning using the same procedure. In this phase, the hidden platform was located in a different quadrant in the pool, diagonal to its previous location. On the day that the criterion for learning was met, or else on day 9 of testing, the platform was removed from the pool, and the group was given a probe trial to evaluate reversal learning.

2.3.7. Marble-burying assay-Adult mice on a B6 background were tested in a Plexiglas cage located in a sound-attenuating chamber with ceiling light and fan. The cage contained corncob bedding $5 \mathrm{~cm}$ deep, with 20 black glass marbles (14 mm diameter) arranged in an equidistant $5 \times 4$ grid on top of the bedding. Animals were given access to the marbles for $30 \mathrm{~min}$. Measures were taken of the number of buried marbles (designated as $2 / 3$ of the marble being covered by the bedding) by an observer blind to genotype.

2.3.8. Contextual and cued fear conditioning-A separate group of B6-background mice were evaluated for learning and memory in a conditioned fear test, using the NearInfrared image tracking system (MED Associates, Burlington, VT). The procedure was conducted across 3 days. On the first day, mice were given a 7-min training session. Mice 
were placed in the test chamber, contained in a sound-attenuating box, and allowed to explore for $2 \mathrm{~min}$. The mice were then exposed to a 30-s tone (90 dB), followed by a 2-s scrambled foot shock $(0.6 \mathrm{~mA})$. Mice received 2 additional shock-tone pairings, with $80 \mathrm{~s}$ between the first and second pairings, and $120 \mathrm{~s}$ between the second and third pairings. Context-dependent learning was evaluated on the second day of testing. Mice were placed back into the original test chamber, and levels of freezing (immobility) were determined across a 5-min session. On the third day of testing, mice were evaluated for associative learning to the auditory cue in a final 5-min session. The conditioning chambers were modified using a Plexiglas insert to change the wall and floor surface, and a novel odor (vanilla flavoring) was added to the sound-attenuating box. Mice were placed in the modified chamber and allowed to explore. After $2 \mathrm{~min}$, the acoustic stimulus was presented for a 3-min period. Levels of freezing before and during the stimulus were obtained by the image tracking system.

\subsection{Statistical analysis}

Data were analyzed using one-way ANOVAs (analysis of variance) or repeated measures ANOVAs, with genotype as a factor. Although two of the experimental groups included both male and female mice, subject numbers were not high enough to include sex as a separate factor in the behavioral analyses. Because of expected group differences, males and females were analyzed separately for body weight. Group means were compared using post hoc Fisher's PLSD (protected least-significant difference) tests only when a significant effect of genotype was found in the overall ANOVA. To determine developmental profiles of body weight in off-spring from our AS breeding colony, we used nonlinear (log-based) regression model comparisons, with separate analyses for males and females from each background strain. Social preference was determined using within-genotype repeated measures ANOVAs, with the factor of chamber side (e.g., stranger side or empty cage side). Similarly, quadrant preference in the Morris water maze was determined using within-genotype repeated measures ANOVAs, with the factor of quadrant location. For all comparisons, significance was set at $p<0.05$.

\section{Results}

\subsection{Weight}

In line with previous reports [14], maternal-deficiency of Ube3a led to significantly increased body weight in almost all of the $U b e 3 a^{m-/ p+}$ groups (Fig. 1). Higher body weights were most pronounced in the female mice on a 129 background [repeated measures ANOVA; genotype main effect, $F(1,10)=7.33, p=0.0221]$, and on a B6 background [genotype $\times$ age interaction, $F(4,44)=13.25, p<0.0001$ ]. The increased body weights were also observed in the male mice on a B6 background [B6 males, cohort 1; genotype $\times$ age interaction, $F(4,40)=4.93, p=0.0025$; B6 males, cohort 2; genotype $\times$ age interaction, $F(4,132)=10.34, p<0.0001]$, but the changes were not consistent across weeks of testing.

To confirm and extend these significant genotype effects, we compiled body weight measures from the juvenile period to late adulthood in an independent set of mice on 129 and B6 backgrounds (Fig. 2). We observed increased body weight in the $U b e 3 a^{m-/ p^{+}}$mice within each sex and background strain [nonlinear regression model comparisons; 129 males, $F(1,131)=52.18, p<0.0001 ; 129$ females, $F(1,119)=22.20, p<0.0001$; B6 males, $F(1,294)=30.84, p<0.0001$; B6 females, $F(1,279)=65.15, p<0.0001]$. The results indicated that maternal $\mathrm{Ube} 3 a$ loss leads to substantial weight gain that becomes progressively apparent with age. 


\subsection{Activity}

Mice were evaluated for locomotion, rearing, and exploration in a 1-h activity test (Fig. 3). In the 129-background group, both $U b e 3 a^{m+/ p+}$ and $U b e 3 a^{m-/ p+}$ mice had generally low levels of locomotion, with almost zero rearing and exploration of the center regions. No significant differences were observed between the 129-background experimental groups. In contrast, significant effects of genotype were found for each of the activity measures in the first B6 cohort. The B6-background $U b e 3 a^{m-/ p+}$ mice had significant decreases in distance traveled [main effect of genotype, $F(1,22)=13.77, p=0.0012$ ] and rearing movements [main effect of genotype, $F(1,22)=25.05, p<0.0001]$. At 14 weeks in age, the B6 $U b e 3 a^{m-/ p+}$ mice spent more time in the center regions than the $U b e 3 a^{m+/ p+}$ mice [genotype $\times$ age interaction, $F(2,44)=5.29, p=0.0087]$. The second $\mathrm{B} 6$ cohort of mice tested in adulthood also demonstrated marked decreases in number of rearing movements $[F(1,33)=$ 23.07, $p<0.0001]$.

\subsection{Wire hang test}

No differences in grip strength were observed in the mice on the 129-background, tested at 7-11 weeks in age, or in the B6-background mice, tested at 5 or 7 weeks in age. In both backgrounds, the wild type and $U b e 3 a^{m-/ p+}$ groups had high levels of proficiency, with the majority of mice remaining on the screen for the entire 60-s trial. However, in the B6background mice tested at 30 weeks in age, the $U b e 3 a^{m-1 p+}$ mice had significantly reduced latencies to fall $(23.8 \mathrm{~s}$ mean, $\mathrm{SEM}=6)$, in comparison to the $U b e 3 a^{m+/ p+}$ mice $(48.0 \mathrm{~s}$ mean, $\mathrm{SEM}=5)[F(1,33)=10.5, p=0.0027]$.

\subsection{Rotarod}

Previous reports $[11,13,14]$ have described deficits in rotarod performance in $U b e 3 a^{m-/ p+}$ mice. In the present study, motor impairment was observed in $U b e 3 a^{m-/ p+}$ mice in both the B6-background cohorts, but not in the 129-background group at the given age (Fig. 4). Overall repeated measures ANOVAs confirmed significant differences in the first cohort of B6 mice [main effect of genotype, $F(1,23)=14.07, p=0.001$ ], and the second cohort of B6 mice [main effect of genotype, $F(1,33)=37.42, p<0.0001$; genotype $\times$ age interaction, $F(8,264)=2.13, p=0.0335]$.

\subsection{Acoustic startle responses}

Decreased startle amplitudes were found in the $U b e 3 a^{m-/ p+}$ group, but only in B6background mice tested during adolescence. The first cohort of B6 $\mathrm{Ube}^{\mathrm{m}} \mathrm{a}^{m-1 p+}$ mice had significantly reduced startle amplitudes when the startle stimulus was administered alone (Fig. 5A), and at every level of the prepulse [post hoc analyses following significant main effect of genotype, $F(1,23)=19.16, p=0.0002$; and genotype $\times$ decibel interaction, $F(6,138)=9.84, p<0.0001]$. This same set of mice also had differences in sensorimotor gating (Fig. 5C). Deficiency of Ube3a led to significant increases in prepulse inhibition in the first $\mathrm{B} 6$ cohort [genotype main effect, $F(1,23)=8.23, p=0.0087$; genotype $\times$ decibel interaction, $F(4,92)=2.84, p=0.0285]$. These age-dependent effects were not determined for the 129-background mice, which were only tested at one time point in young adulthood.

\subsection{Sociability in a 3-chamber choice task}

Allensworth et al. [23], using a 3-chamber task, found that adult, B6-background $U b e 3 a^{m-/ p+}$ mice have significant reductions in exploration, but no changes in social approach. The present study confirmed these findings in adolescent B6-background mice, which showed no significant genotype differences in time spent in each side of the test box or time spent sniffing the wire cages (Fig. 6). However, only the control mice demonstrated a significant preference for spending more time in the side with the stranger mouse, versus 
the empty cage side [within-genotype comparisons following significant main effect of side, $F(1,23)=5.05, p=0.0346]$. Both genotypes demonstrated a significant preference for sniffing the stranger mouse cage, in comparison to the empty cage [within-genotype comparisons following significant main effect of side, $F(1,23)=18.93, p=0.0002]$. Similar to the findings of Allensworth et al. [23], the $U b e 3 a^{m-/ p+}$ mice had significantly reduced exploration during the social approach test, measured by entries into the two side chambers [genotype main effect, $F(1,23)=11.09, p=0.0029$ ].

The present study also examined social approach in the 129-background mice.

Unfortunately, the two genotypes had markedly low activity in the 3-chamber apparatus, with 70\% (7/10) of the $U b e 3 a^{m+/ p+}$ mice and 55\% (6/11) of the Ube $3 a^{m-/ p+}$ mice having zero entry scores for one or both of the side chambers. This failure to explore in the choice task precluded meaningful interpretations of social behavior scores in the 129 group.

\subsection{Morris water maze}

3.7.1. Visual cue task-Although maternal $U b e 3 a$ deletion did not lead to overt deficits in the ability to locate a cued visible platform (Table 2), $U b e 3 a^{m-/ p+}$ mice from both background strains showed lower swim velocity on one or two days of testing [129background, genotype $\times$ day interaction, $F(1,19)=4.58, p=0.0456$ ]; B6 (cohort 1), main effect of genotype, $F(1,23)=19.98, p=0.0002$; and B6 (cohort 2), main effect of genotype, $F(1,33)=20.34, p<0.0001$; genotype $\times$ day interaction, $F(1,33)=12.17, p=0.0014$. Only the 129-background mice had genotype differences in latency to reach the escape platform [genotype $\times$ day interaction; $F(1,19)=4.44, p=0.0487$ ]. Post hoc tests indicated that, although the $U b e 3 a^{m-/ p+}$ mice in the 129 group had longer latencies on day 1 of testing, these differences were no longer present by day 2 . These data provide evidence that, overall, the $U b e 3 a^{m-/ p+}$ mice had reduced swimming ability, but could still achieve escape latencies similar to the $U b e 3 a^{m+/ p+}$ mice.

3.7.2. Acquisition and reversal in the Morris water maze-Previous work has shown that loss of Ube3a function in B6- or mixed 129/B6-background mice can lead to impaired spatial learning in a water maze task $[13,14]$. In the 129-background mice of the present study, the $U b e 3 a^{m-/ p+}$ mice had only subtle changes in acquisition, but overt deficits in reversal learning (Fig. 7A and B). During the acquisition phase, both the $U b e 3 a^{m+/ p+}$ and $U b e 3 a^{m-/ p+}$ mice reached the 15 -s criterion for learning. Although a repeated measures ANOVA indicated a significant genotype $\times$ day interaction for acquisition $[F(5,95)=2.78, p$ $=0.022]$, post hoc tests did not indicate significant group differences on any one day of testing. However, during reversal learning, the $129 \mathrm{Ube} 3 \mathrm{a}^{\mathrm{m}-\mathrm{pt}}$ mice never reached criterion for learning, and demonstrated significantly longer latencies to the escape platform, in comparison to the wild type mice, on almost every test day [post hoc tests following main effect of genotype, $F(1,19)=18.15, p=0.0004]$.

In the B6-background mice, learning in the Morris water maze was dependent upon age at testing. At 8 weeks of age, Ube $3 a^{m+/ p+}$ and $U b e 3 a^{m-/ p+}$ mice in the first B6 cohort had similar performance during acquisition of the spatial learning task (Fig. $7 \mathrm{C}$ and D). During reversal learning, there was a non-significant trend for the $U b e 3 a^{m-/ p+}$ mice in the first cohort to have longer latencies to reach the escape platform [main effect of genotype, $F(1,23)=4.26, p=0.0506]$. However, in the B6 cohort tested at 16 weeks of age, the $U b e 3 a^{m-/ p+}$ mice demonstrated marked deficits in acquisition, with significantly longer escape latencies than the $U b e 3 a^{m+/ p+}$ mice across most days of testing [main effect of genotype, $F(1,19)=19.67, p=0.0003]$. Because of this impairment in the initial training phase, the older B6 mice were not further tested in the reversal learning task. 
3.7.3. Quadrant selectivity in platform crosses-The results from the 1-min probe tests with the platform removed confirmed selective deficits in reversal learning in the 129background mice (Fig. 8A and B). While there were no group differences in the probe trial following acquisition, significant effects of genotype emerged after reversal learning [repeated measure ANOVA for target and opposite quadrant; main effect of genotype, $F(1,19)=9.46, p=0.0062$; genotype $\times$ target location interaction, $F(1,19)=6.15, p=$ $0.0227]$. During acquisition, within-genotype repeated measures ANOVAs revealed strong target selectivity across all four quadrants in both groups [wild type, $F(3,27)=19.23, p<$ $0.0001 ; \mathrm{m}-1 \mathrm{p}+, F(3,30)=20.62, p<0.0001]$. However, during reversal learning, only the Ube $3 a^{m+/ p+}$ mice demonstrated a significant preference for the new platform location $[F(3,27)=40.06, p<0.0001]$. The $U b e 3 a^{m-p+}$ mice did not exhibit the same spatial preference, even after 9 days of training in the reversal task.

A different trend was observed in the first cohort of B6-background mice tested at 8 weeks of age (Fig. 8C and D). Following both phases of testing, only the $U b e 3 a^{m+/ p+}$ mice demonstrated significant preference for the platform location in the target quadrant [acquisition, $F(3,39)=4.6, p=0.0075$; reversal, $F(3,39)=6.33, p=0.0013$ ]. While there were no group differences in the probe trial following acquisition, significant effects of genotype emerged after reversal learning [main effect of genotype, $F(1,23)=10.46, p=$ 0.0037]. In the second cohort of B6-background mice, neither genotype demonstrated a strong preference for the target location, versus the other three quadrant locations (Fig. 8E). However, the $U b e 3 a^{m-p+}$ mice had significantly fewer crossings over the target location [main effect of genotype, $F(1,19)=20.46, p=0.0002$ ]. These findings from the probe trials suggest that the deficiency in Ube3a led to a failure to develop target selectivity in the B6background mice, even in the case when escape latencies were similar to that of controls.

\subsection{Marble-burying assay}

The second cohort of B6-background mice was also assessed, at the age of 33 weeks, for digging and response to novelty in a marble-burying assay (Fig. 9). Highly significant genotype differences were observed in the number of marbles covered by bedding in the 30min task. Ube $3 a^{m-/ p+}$ mice buried significantly fewer marbles, in comparison to the Ube $3 a^{m+/ p+}$ mice $[F(1,33)=44.57, p<0.0001]$. These overt deficits in digging behavior were confirmed in a younger cohort of male mice, given two marble-burying tests, one week apart $[F(1,16)=32.55, p<0.0001]$.

\subsection{Contextual and cue fear conditioning}

A potential caveat of studies using the Morris water maze task is that the evaluation of learning can be confounded by deficits in swimming ability. Therefore, a separate cohort of B6-background male mice was evaluated in a conditioned fear task, which does not require the same degree of motor skills. Previous studies have reported that $U b e 3 a^{m-/ p+}$ mice on a hybrid 129/B6 background have deficits in context-dependent, but not cue-dependent, learning $[11,14]$. A different pattern of altered fear conditioning emerged in the present study (Fig. 10). The Ube3a $a^{m-p+}$ mice on a B6-background did not demonstrate impaired contextual fear conditioning [non-significant main effect of genotype, $F(1,23)=3.59, p=$ 0.0708]. However, during the test for cue-dependent learning, the Ube $3 a^{m-1 p+}$ mice had significant decreases in freezing behavior during the first two minutes of the auditory cue [main effect of genotype; $F(1,23)=4.71, p=0.0406$; and genotype $\times$ time interaction, $F(4,92)=3.14, p=0.0182]$. These findings provide further confirmation that $U b e 3 a$ dysfunction leads to learning and memory deficits. 


\section{Discussion}

The present studies demonstrate that AS-like behavioral phenotypes in $U b e 3 a^{m-1 p+}$ mice can vary across genetic background, age at testing, and cohort group. In particular, Ube $3 a^{m-1 p+}$ mice on the B6 background exhibited motor deficits and impaired acquisition of spatial learning, dependent on age and cohort. Marked deficits in spatial learning acquisition were found in B6 mice at 16 weeks of age, but not at 8 weeks of age, suggesting progressive loss of cognitive function across time. In contrast, alterations in startle reactivity and sensorimotor gating were only observed in adolescent B6 mice, but not adult B6 mice, indicating a limited developmental window for investigating some abnormal phenotypes in the AS model. One caveat to these findings is that possible differences between alterations in male and female AS mice were not determined.

In our study, the B6 Ube $3 a^{m-/ p+}$ mice had impaired motor coordination on a rotarod and deficits in acquisition of spatial learning, in line with a previous report on behavioral abnormalities in $U b e 3 a^{m-/ p+}$ mice on a C57BL/6J background [13]. Similarly, AS-model mice on a predominantly B6 background have deficits in rotarod performance, grip strength, and other motor tasks [24]. Overt rotarod impairment and a lack of quadrant preference in the water maze have also been found in $U b e 3 a^{m-1 p+}$ mice on a hybrid 129/B6 background $[11,14]$. In the present studies, $U b e 3 a^{m-/ p+}$ mice on a 129-background had no changes in rotarod performance at ages 8-13 weeks, and only subtle changes in acquisition in the water maze, but these mice had marked impairment in reversal learning. Generally low levels of activity in the 129 mice made the detection of changes in exploration or social preference difficult. Overall, our findings support the use of the $U b e 3 a^{m-/ p+}$ mice on a B6 background for behavioral rescue studies targeting activity and motor coordination, or either 129- or B6background mice for studies focused on deficits in cognitive function.

Reversal learning in the water maze has been used as a measure of behavioral flexibility and perseveration in mice, relevant to the cognitive rigidity and resistance to change associated with autism and other neurodevelopmental disorders [20,25]. Studies in children with Down syndrome, fragile $\mathrm{X}$ syndrome, and autism have reported specific deficits in the ability to change learned patterns of behavior during spatial-reversal or set-shifting reversal tasks [2629]. Similar deficits in reversal learning have been reported for mice with targeted disruption of Nrcam, an autism candidate gene [30], or Fmrl, the gene underlying fragile X syndrome [31-34]. The deficits in reversal learning observed in the present study could reflect selective impairment in cognitive flexibility and lack of adaptability in $U b e 3 a^{m-1 p^{+}}$ mice.

One goal of the present study was to identify specific phenotypes that would be optimal targets in preclinical drug screens for AS intervention. From approximately 7 to 31 weeks in age, $U b e 3 a^{m-/ p+}$ mice on a B6 background consistently showed significant deficits in rearing during an activity test and coordination on an accelerating rotarod, suggesting that these measures would be particularly valuable for tracking rescue of motor phenotypes from adolescence through adulthood. Although the marble-burying task was only conducted in adult mice (from 18 to 33 weeks in age), the differences between the wild type and Ube3adeficient mice were highly significant, suggesting possible utility of this relatively simple and quick procedure. Our results also support the use of the conditioned fear test in B6 $U b e 3 a^{m-/ p+}$ mice. Given the past reports of significant deficits in contextual fear conditioning $[11,13,14]$, and more recent findings of impaired cue-dependent fear conditioning ([15]; present study), there is good justification for conducting both phases of the learning procedure. 
An advantage of the activity, rotarod, and marble-burying assays is that performance can be assessed following acute drug treatments. Similarly, in the conditioned fear procedure, a single drug administration can be given immediately after the initial training (the shockexposure phase), with evaluation of drug efficacy against learning deficits occurring hours or days later. For example, Kaphzan et al. [12] investigated whether decreasing neuregulin 1 (Nrg1) signaling through the ErbB4 receptor could reverse cognitive impairment in ASmodel mice. The researchers found that an ErbB inhibitor, administered directly following training, could rescue conditioned fear deficits in AS-model mice during contextual tests given 7 days and, to a lesser extent, 36 days later. For a broader evaluation of therapeutic efficacy in cognitive impairment, testing mice for acquisition and reversal learning in the Morris water maze would allow assessment of drug effects on rates of learning across time and detrimental cognitive rigidity. Because the water maze assays require multiple days of training, these procedures would be especially appropriate to investigate chronic dosing regimens, persistent effects of early intervention, and rescue through genetic alterations.

One limitation of our studies was that the number of subjects was not sufficient to determine possible sex-dependent effects of the maternal Ube3a deletion. However, previous work has shown that significant alterations in behavior, such as motor deficits and impaired spatial learning, can be observed in mixed-sex groups of $U b e 3 a^{m-/ p+}$ mice [14]. Similarly, we found that some abnormal phenotypes, including reduced rearing and shorter latencies to fall from the rotarod, were apparent in both mixed-sex and all-male groups of AS-model mice. Although our findings suggest male and female mice can be combined for experimental groups, further work is needed to confirm that both sexes have comparable vulnerability to AS-like behavior, and whether abnormal phenotypes in $U b e 3 a^{m-/ p+}$ female mice vary with phase of estrous cycle.

In conclusion, the present study determined extensive behavioral profiles of the $U b e 3 a^{m-/ p+}$ mice on 129- and B6-background strains, and identified several functional targets for the evaluation of novel therapeutics for AS, such as recently discovered topoisomerase inhibitors for unsilencing Ube3a [16]. Aberrant phenotypes found in $U b e 3 a^{m-/ p+}$ mice included decreased rearing movements, grip strength, and marble-burying, impaired motor coordination, and deficits in spatial, reversal, and conditioned fear learning. Inconsistency of some behavioral alterations, such as hypoactivity or deficient rotarod performance, across sets of AS-model mice provided evidence for the importance of background strain, age, previous testing experience, and possibly sex in changes arising from $U b e 3 a^{m-/ p+}$ maternal deletion.

\section{Acknowledgments}

The authors wish to thank Dr. Yong-hui Jiang for his kind generosity in providing Ube3a breeding pairs for our colony. This work was supported by the Simons Foundation, the Angelman Syndrome Foundation, NIMH grant 1R01MH093372 to BDP, and NICHD grant P30HD03110 to Dr. Joe Piven. These sponsors did not have involvement in study design, data collection, analysis, or interpretation, writing the report, or decision to submit the article for publication.

\section{References}

1. Angelman H. "Puppet" children. A report on three cases. Developmental Medicine and Child Neurology. 1965; 7:681-8.

2. Mabb AM, Judson MC, Zylka MJ, Philpot BD. Angelman syndrome: insights into genomic imprinting and neurodevelopmental phenotypes. Trends in Neuro-sciences. 2011; 34:293-303.

3. Matsuura T, Sutcliffe JS, Fang P, Galjaard RJ, Jiang YH, Benton CS, et al. De novo truncating mutations in E6-AP ubiquitin-protein ligase gene (UBE3A) in Angelman syndrome. Nature Genetics. 1997; 15:74-7. [PubMed: 8988172] 
4. Kishino T, Lalande M, Wagstaff J. UBE3A/E6-AP mutations cause Angelman syndrome. Nature Genetics. 1997; 15:70-3. [PubMed: 8988171]

5. Albrecht U, Sutcliffe JS, Cattanach BM, Beechey CV, Armstrong D, Eichele G, et al. Imprinted expression of the murine Angelman syndrome gene, Ube3a, in hippocampal and Purkinje neurons. Nature Genetics. 1997; 17:75-8. [PubMed: 9288101]

6. Rougeulle C, Glatt H, Lalande M. The Angelman syndrome candidate gene, UBE3A/E6-AP, is imprinted in brain. Nature Genetics. 1997; 17:14-5. [PubMed: 9288088]

7. Yashiro K, Riday TT, Condon KH, Roberts AC, Bernardo DR, Prakash R, et al. Ube3a is required for experience-dependent maturation of the neocortex. Nature Neuroscience. 2009; 12:777-83.

8. Sato M, Stryker MP. Genomic imprinting of experience-dependent cortical plasticity by the ubiquitin ligase gene Ube3a. Proceedings of the National Academy of Sciences of the United States of America. 2010; 107:5611-6. [PubMed: 20212164]

9. Dindot SV, Antalffy BA, Bhattacharjee MB, Beaudet AL. The Angelman syndrome ubiquitin ligase localizes to the synapse and nucleus, and maternal deficiency results in abnormal dendritic spine morphology. Human Molecular Genetics. 2008; 17:111-8. [PubMed: 17940072]

10. Wallace ML, Burette AC, Weinberg RJ, Philpot BD. Maternal loss of Ube3a produces an excitatory/inhibitory imbalance through neuron type-specific synaptic defects. Neuron. 2012; 74:793-800. [PubMed: 22681684]

11. Jiang YH, Armstrong D, Albrecht U, Atkins CM, Noebels JL, Eichele G, et al. Mutation of the Angelman ubiquitin ligase in mice causes increased cytoplasmic p53 and deficits of contextual learning and long-term potentiation. Neuron. 1998; 21:799-811. [PubMed: 9808466]

12. Kaphzan H, Hernandez P, Jung JI, Cowansage KK, Deinhardt K, Chao MV, et al. Reversal of impaired hippocampal long-term potentiation and contextual fear memory deficits in Angelman syndrome model mice by ErbB inhibitors. Biological Psychiatry. 2012; 72:182-90. [PubMed: 22381732]

13. Miura K, Kishino T, Li E, Webber H, Dikkes P, Holmes GL, et al. Neurobehavioral and electroencephalographic abnormalities in Ube3a maternal-deficient mice. Neurobiology of Disease. 2002; 9:149-59. [PubMed: 11895368]

14. van Woerden GM, Harris KD, Hojjati MR, Gustin RM, Qiu S, de Avila Freire R, et al. Rescue of neurological deficits in a mouse model for Angelman syndrome by reduction of alphaCaMKII inhibitory phosphorylation. Nature Neuroscience. 2007; 10:280-2.

15. Baudry M, Kramar E, Xu X, Zadran H, Moreno S, Lynch G, et al. Ampakines promote spine actin polymerization, long-term potentiation, and learning in a mouse model of Angelman syndrome. Neurobiology of Disease. 2012; 47:210-5. [PubMed: 22525571]

16. Huang HS, Allen JA, Mabb AM, King IF, Miriyala J, Taylor-Blake B, et al. Topoisomerase inhibitors unsilence the dormant allele of Ube3a in neurons. Nature. 2012; 481:185-9.

17. Crabbe JC, Wahlsten D, Dudek BC. Genetics of mouse behavior: interactions with laboratory environment. Science. 1999; 284:1670-2. [PubMed: 10356397]

18. Gentile JK, Tan WH, Horowitz LT, Bacino CA, Skinner SA, Barbieri-Welge R, et al. A neurodevelopmental survey of Angelman syndrome with genotype-phenotype correlations. Journal of Developmental and Behavioral Pediatrics. 2010; 31:592-601. [PubMed: 20729760]

19. Paylor R, Crawley JN. Inbred strain differences in prepulse inhibition of the mouse startle response. Psychopharmacology. 1997; 132:169-80. [PubMed: 9266614]

20. Moy SS, Nadler JJ, Young NB, Perez A, Holloway LP, Barbaro RP, et al. Mouse behavioral tasks relevant to autism: phenotypes of 10 inbred strains. Behavioural Brain Research. 2007; 176:4-20. [PubMed: 16971002]

21. Nadler JJ, Moy SS, Dold G, Trang D, Simmons N, Perez A, et al. Automated apparatus for quantitation of social approach behaviors in mice. Genes, Brain, and Behavior. 2004; 3:303-14.

22. Morris R. Developments of a water-maze procedure for studying spatial learning in the rat. Journal of Neuroscience Methods. 1984; 11:47-60. [PubMed: 6471907]

23. Allensworth M, Saha A, Reiter LT, Heck DH. Normal social seeking behavior, hypoactivity and reduced exploratory range in a mouse model of Angelman syndrome. BMC Genetics. 2011; 12:7. [PubMed: 21235769] 
24. Heck DH, Zhao Y, Roy S, LeDoux MS, Reiter LT. Analysis of cerebellar function in Ube3adeficient mice reveals novel genotype-specific behaviors. Human Molecular Genetics. 2008; 17:2181-9. [PubMed: 18413322]

25. Moy SS, Nadler JJ. Advances in behavioral genetics: mouse models of autism. Molecular Psychiatry. 2008; 13:4-26. [PubMed: 17848915]

26. Coldren JT, Halloran C. Spatial reversal as a measure of executive functioning in children with autism. Journal of Genetic Psychology. 2003; 164:29-41. [PubMed: 12693742]

27. Kogan CS, Boutet I, Cornish K, Graham GE, Berry-Kravis E, Drouin A, et al. A comparative neuropsychological test battery differentiates cognitive signatures of Fragile $\mathrm{X}$ and Down syndrome. Journal of Intellectual Disability Research. 2009; 53:125-42. [PubMed: 19054268]

28. Van der Molen MJ, Van der Molen MW, Ridderinkhof KR, Hamel BC, Curfs LM, Ramakers GJ. Attentional set-shifting in fragile $\mathrm{X}$ syndrome. Brain and Cognition. 2012; 78:206-17. [PubMed: 22261226]

29. Yerys BE, Wallace GL, Harrison B, Celano MJ, Giedd JN, Kenworthy LE. Set-shifting in children with autism spectrum disorders: reversal shifting deficits on the Intradimensional/ Extradimensional Shift Test correlate with repetitive behaviors. Autism. 2009; 13:523-38. [PubMed: 19759065]

30. Moy SS, Nonneman RJ, Young NB, Demyanenko GP, Maness PF. Impaired sociability and cognitive function in Nrcam-null mice. Behavioural Brain Research. 2009; 205:123-31. [PubMed: 19540269]

31. Bakker CEVC, Willemsen R, van der Helm R, Oerlemans F, Vermey M, Bygrave A, et al. Fmr1 knockout mice: a model to study fragile $\mathrm{X}$ mental retardation. The Dutch-Belgian Fragile $\mathrm{X}$ Consortium. Cell. 1994; 78:23-33. [PubMed: 8033209]

32. Kooy RF, D'Hooge R, Reyniers E, Bakker CE, Nagels G, De Boulle K, et al. Transgenic mouse model for the fragile X syndrome. American Journal of Medical Genetics. 1996; 64:241-5. [PubMed: 8844056]

33. Paradee W, Melikian HE, Rasmussen DL, Kenneson A, Conn PJ, Warren ST. Fragile X mouse: strain effects of knockout phenotype and evidence suggesting deficient amygdala function. Neuroscience. 1999; 94:185-92. [PubMed: 10613508]

34. Van Dam D, D'Hooge R, Hauben E, Reyniers E, Gantois I, Bakker CE, et al. Spatial learning, contextual fear conditioning and conditioned emotional response in Fmr1 knockout mice. Behavioural Brain Research. 2000; 117:127-36. [PubMed: 11099766] 


\section{HIGHLIGHTS}

Ube $3 a^{m-/ p+}$ mice had impaired reversal learning in the Morris water maze.

- Deficient acquisition of spatial learning varied across background strain and age.

Aberrant phenotypes included deficits in rearing, rotarod ability, and marbleburying.

- The C57BL/6J background conferred susceptibility to a range of abnormal behaviors. 


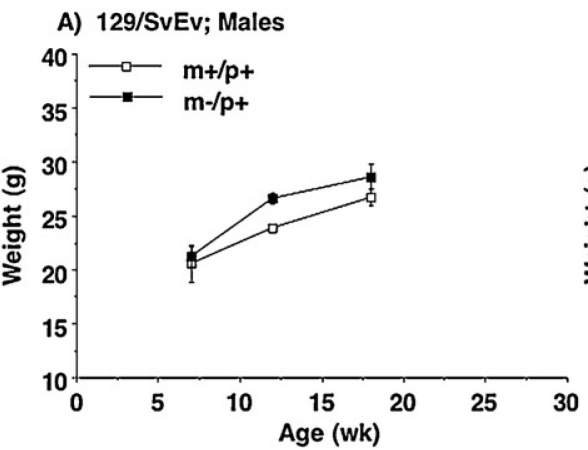

B) $129 / \mathrm{SvEv}$; Females

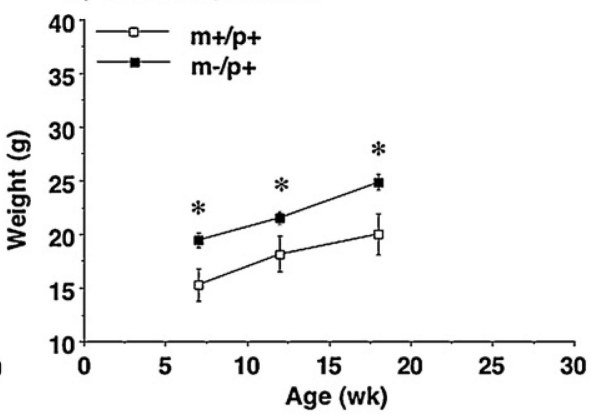

D) C57BL/6J, Cohort 1; Females

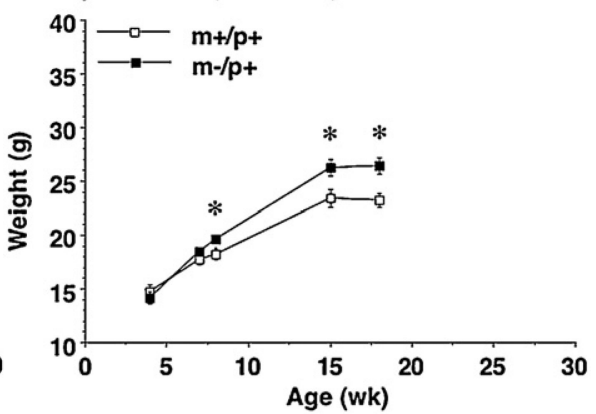

E) C57BL6J, Cohort 2; Males

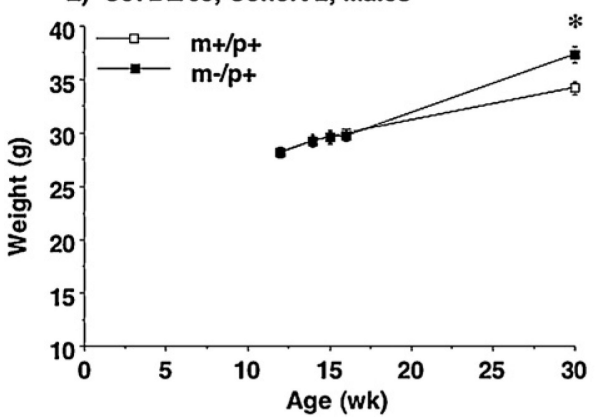

Fig. 1.

Increased body weight in $U b e 3 a^{m-/ p+}$ mice. Data shown are means $( \pm \mathrm{SEM})$ for three separate cohort groups: mice on a $129 \mathrm{~S} 7 / \mathrm{SvEvBrd}-\mathrm{Hprt}^{b-m 2}(129 / \mathrm{SvEv})$ background (A and $\mathrm{B})$, mice on a C57BL/6J background that started testing during adolescence (C and D), and mice on a $\mathrm{C} 57 \mathrm{BL} / 6 \mathrm{~J}$ background that started testing during adulthood $(\mathrm{E})$. Ages are approximate number of weeks. $* p<0.05$. 
A) $129 / \mathrm{SvEv}$; Males

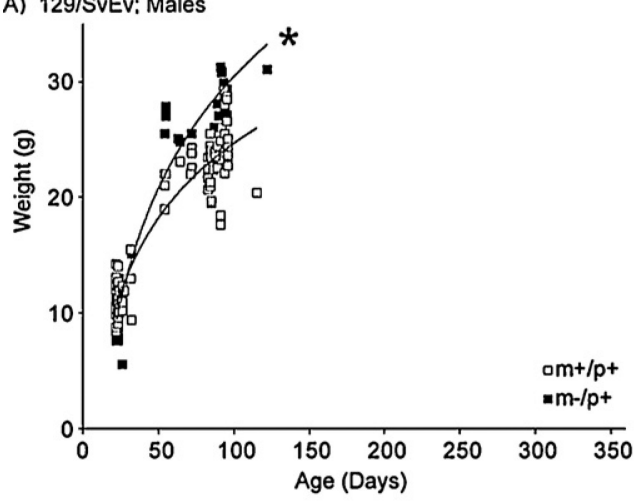

C) C57BL/6J; Males

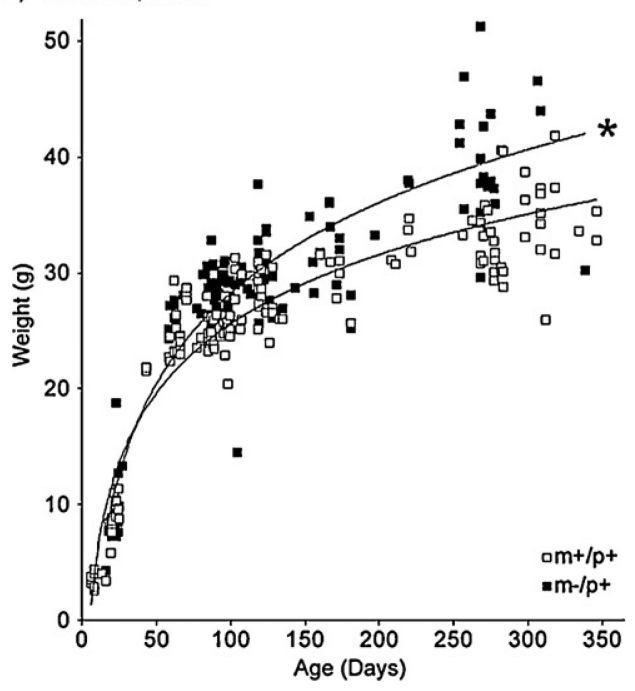

B) $129 / \mathrm{SvEv}$ : Females

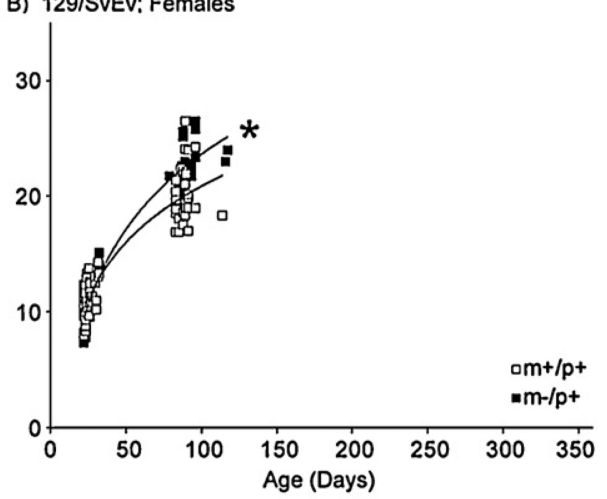

D) C57BL/6J; Females

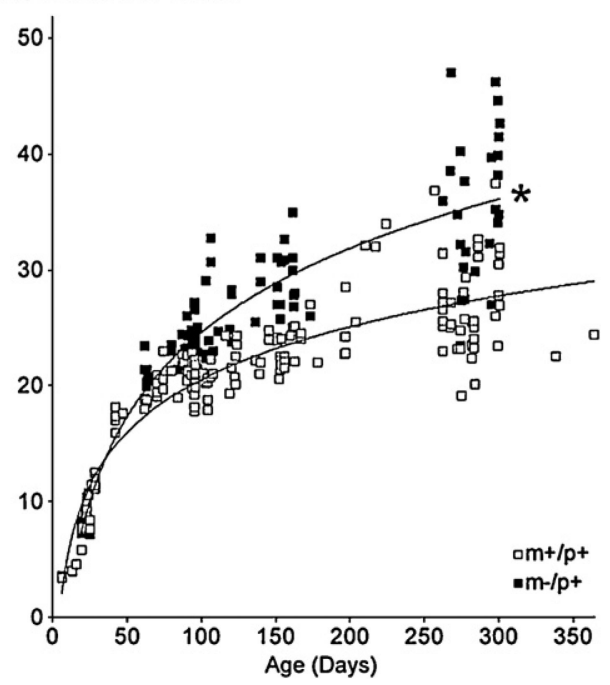

Fig. 2.

Developmental profiles of body weight in $U b e 3 a^{m+/ p+}$ and $U b e 3 a^{m-/ p+}$ mice. Data shown are scatter plots with fitted logarithmic regression lines. Mice were offspring from the same breeding colony that provided subjects for the behavioral studies. Groups included mice on a 129S7/SvEvBrd-Hprt ${ }^{b-m 2}$ (129/SvEv) background (A and B) and C57BL/6J background (C and $\mathrm{D})$, with one weight measure per subject. $* p<0.0001$, nonlinear regression model comparison. 
A) Distance Traveled

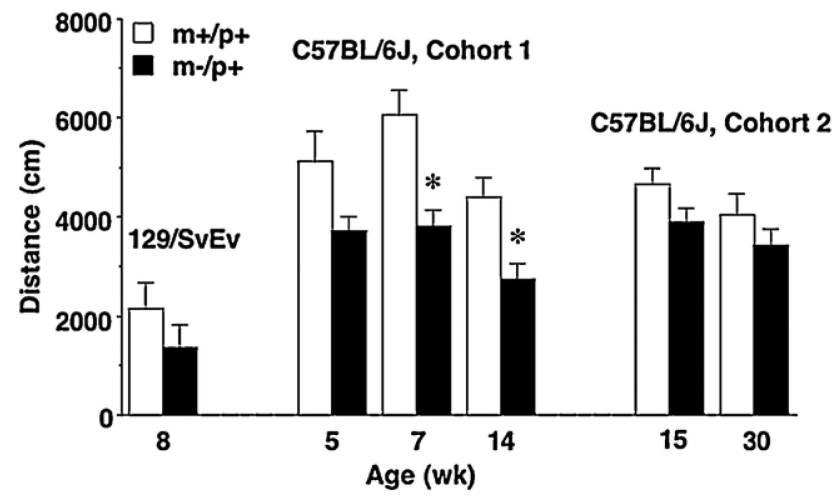

B) Rearing Movements

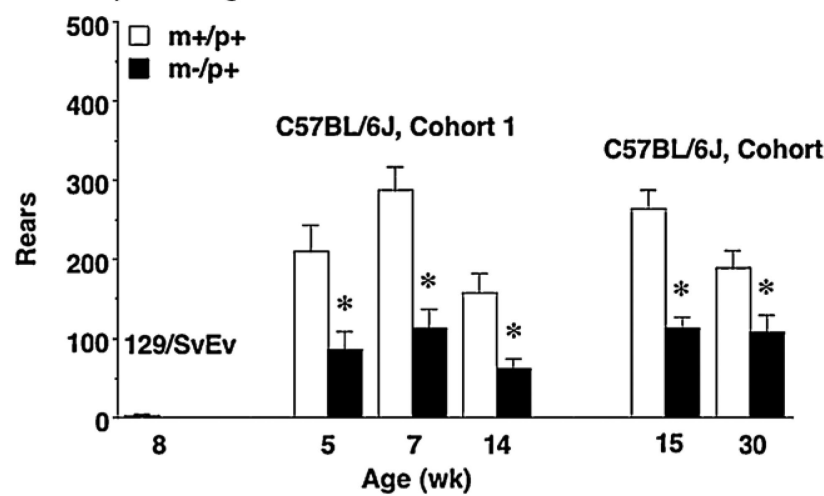

C) Time in Center Region

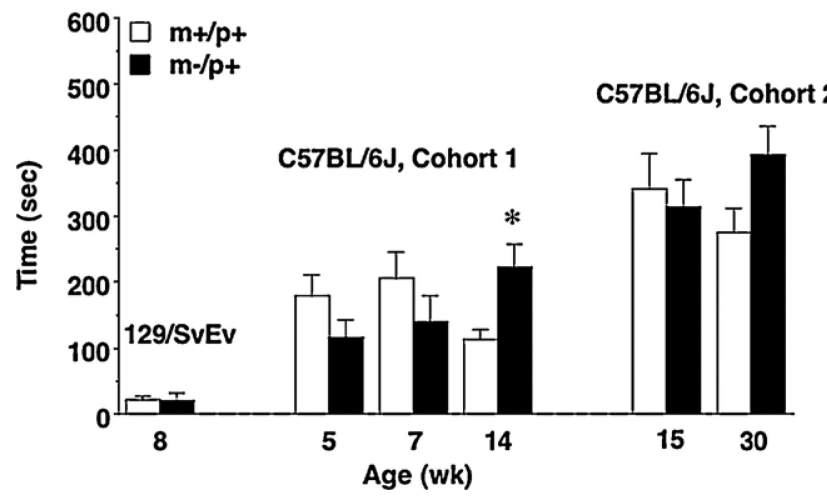

Fig. 3.

Locomotion, rearing, and exploration during an activity test. The $U b e 3 a^{m-/ p+}$ mice on a C57BL/6J background had significant decreases in rearing movements at every time point. $129 /$ SvEv-background mice had generally low activity during the test. Data shown are means $(+\mathrm{SEM})$ for each group for a 1 -h session. One mouse from the $\mathrm{m}-\mathrm{p}+$ group in cohort 1 of the C57BL/6J-background mice was not tested at the 7-week time point due to experimenter error. ${ }^{*} p<0.05$. 
A) $129 / \mathrm{SvEv}$

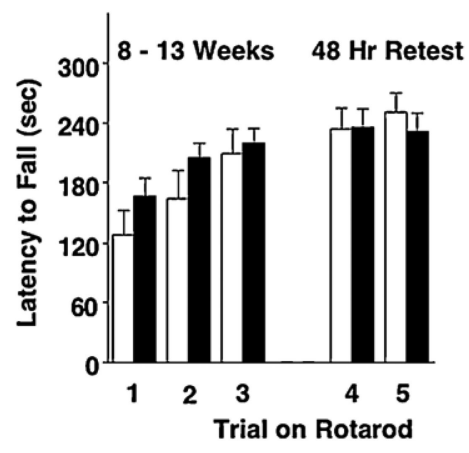

B) C57BL/6J, Cohort 1

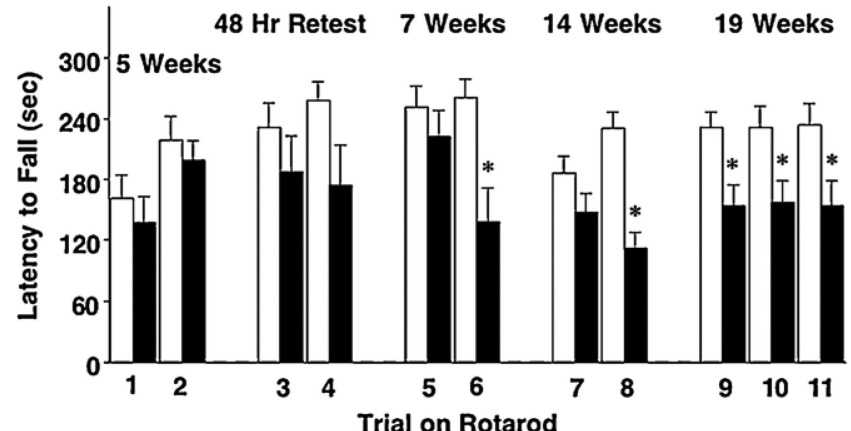

C) C57BL/6J, Cohort 2

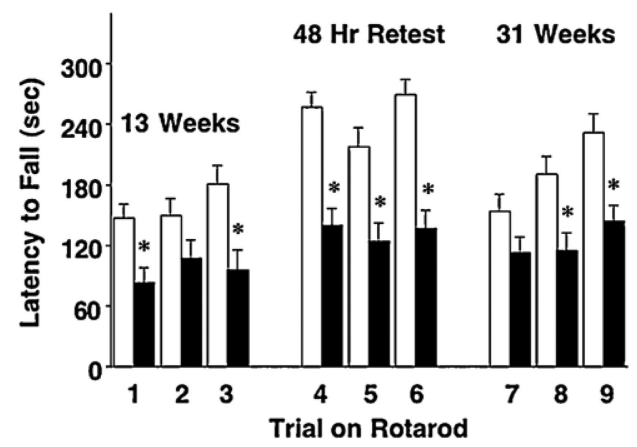

Fig. 4.

Latency to fall from an accelerating rotarod. Mice were given a retest $48 \mathrm{~h}$ following the initial acquisition trials. Deficits were observed in the $U b e 3 a^{m-/ p+}$ groups on a C57BL/6J background. Data shown are means (+SEM). ${ }^{*} p<0.05$. 
A) Amplitude of Acoustic Startle Responses

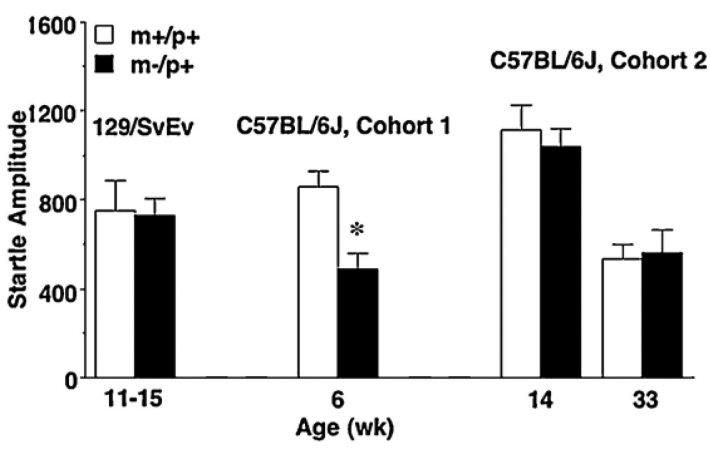

B) 129/SvEv (11-15 Weeks)

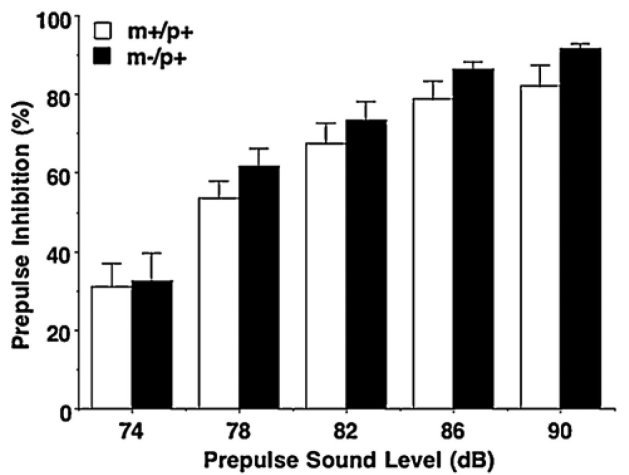

D) C57BL/6J, Cohort 2 (14 Weeks)

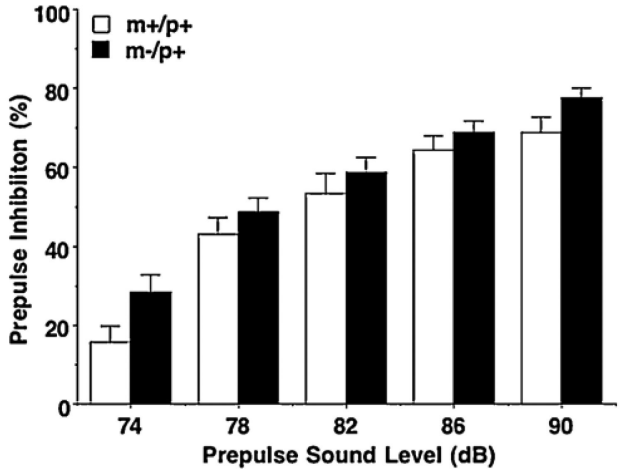

C) C57BL6J, Cohort 1 (6 Weeks)

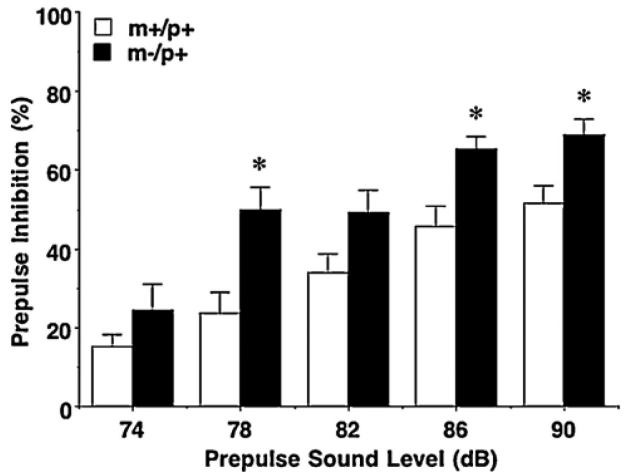

E) C57BL/6J, Cohort 2 (33 Weeks)

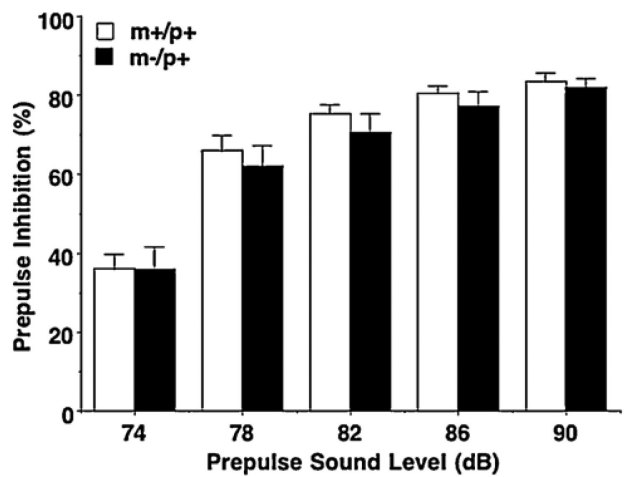

Fig. 5.

Prepulse inhibition of acoustic startle responses. The $U b e 3 a^{m-/ p+}$ mice, on a B 6 background, had significantly reduced startle magnitude following a 120 decibel acoustic stimulus (A) and enhanced prepulse inhibition (C) at 6 weeks of age. Data shown are means $(+\mathrm{SEM}) .{ }^{*} p$ $<0.05$. 
A) Time Spent in Each Side

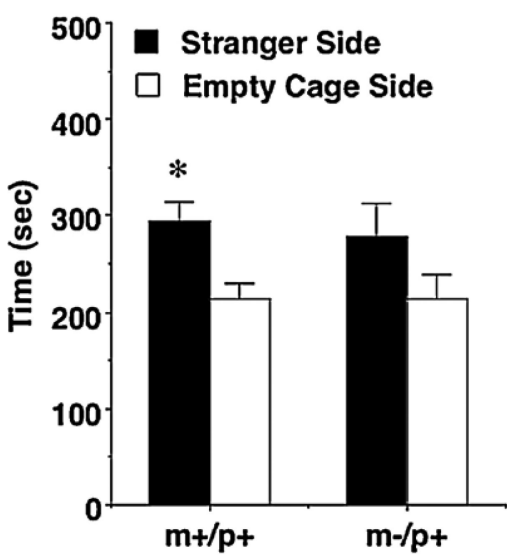

B) Time Sniffing Each Cage

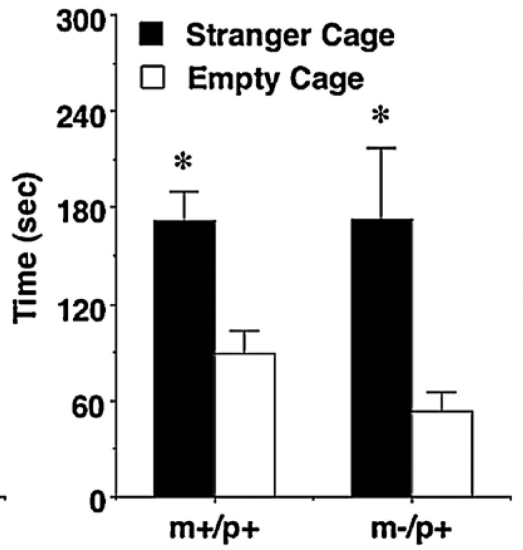

C) Entries into Each Side

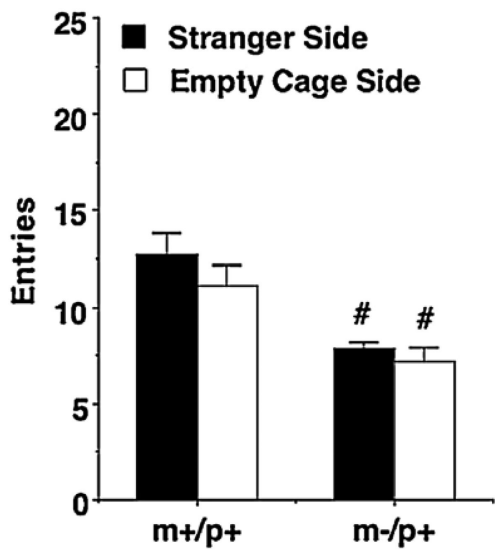

Fig. 6.

Sociability in a three-chamber choice task. Mice were on a C57BL/6J background, and were tested at 6 weeks of age. The $U b e 3 a^{m-/ p+}$ group exhibited significantly reduced exploration during the test. Data shown are mean $(+\mathrm{SEM})$ for a 10 -min test. $* p<0.05$, within-group comparison between the stranger side and empty cage side. ${ }^{\#} p<0.05$, comparison with Ube $3 a^{m+/ p+}$ group. 
A) Acquisition; 129/SvEv (19-22 Weeks)

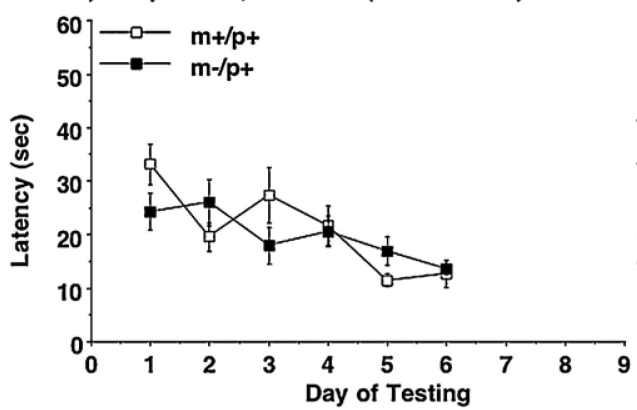

C) Acquisition; C57BL6J (Cohort 1, 8 Weeks)

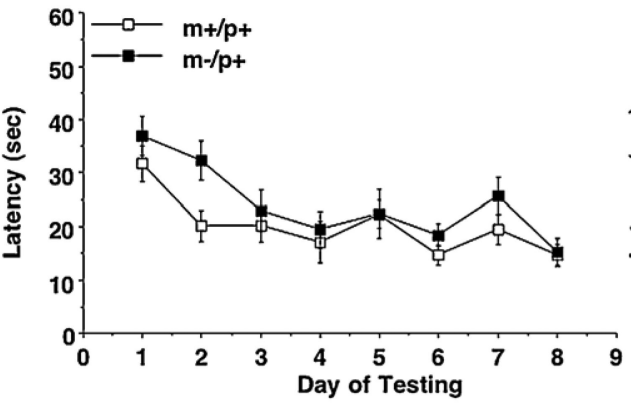

E) Acquisition; C57BL6J (Cohort 2, 17 Weeks)

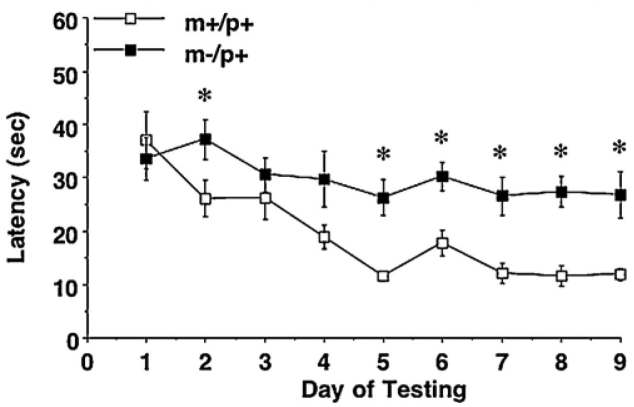

B) Reversal; 129/SvEv (20-23 Weeks)

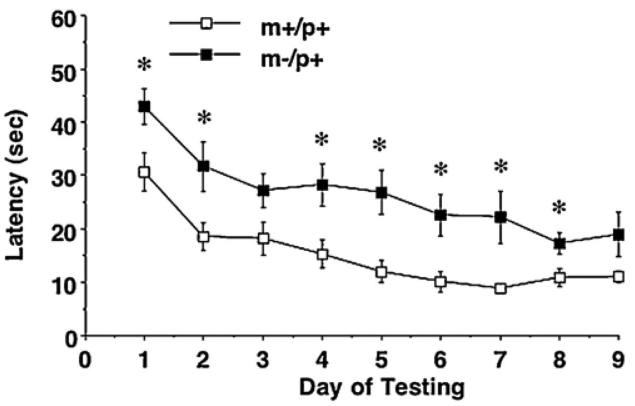

D) Reversal; C57BL/6J (Cohort 1, 9 Weeks)

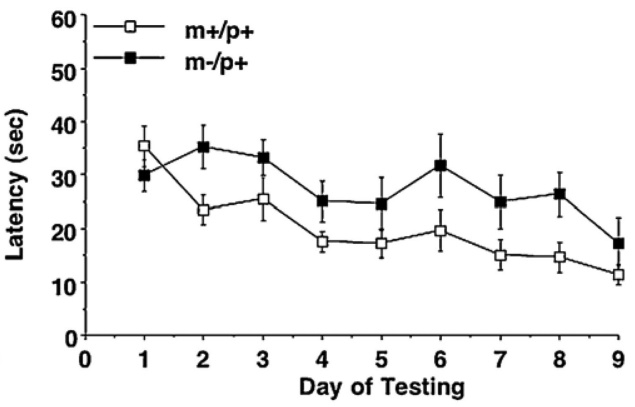

Fig. 7.

Acquisition and reversal of spatial learning in the Morris water maze. During the reversal phase, the location of the escape platform was moved to the opposite quadrant of the maze from the location during training. Data shown are mean $( \pm \mathrm{SEM})$ of four trials per day. ${ }^{*} p<$ 0.05 . 
A) $129 / \mathrm{SvEv}$ (19-22 weeks)

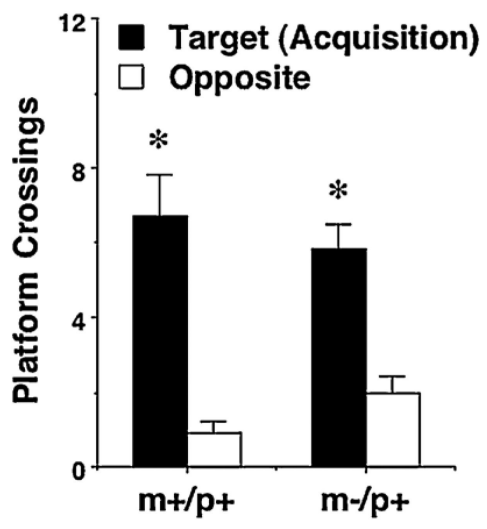

C) C57BL/6J (Cohort 1, 8 Weeks)

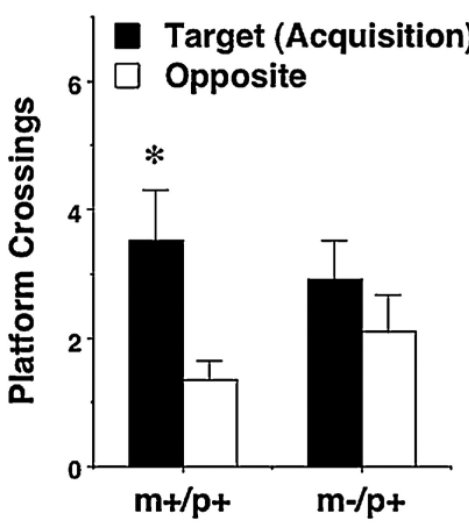

E) C57BL/6J (Cohort 2, 17 Weeks)

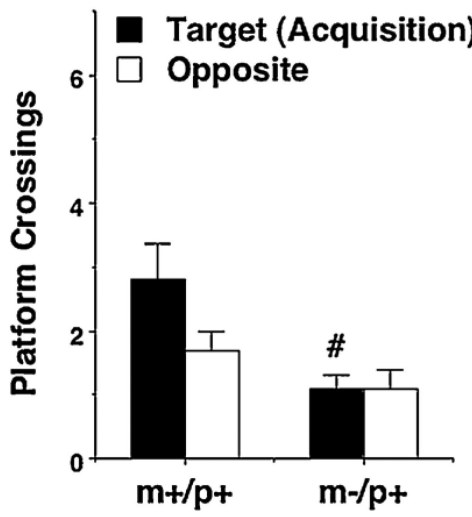

B) 129/SvEv (20-23 weeks)

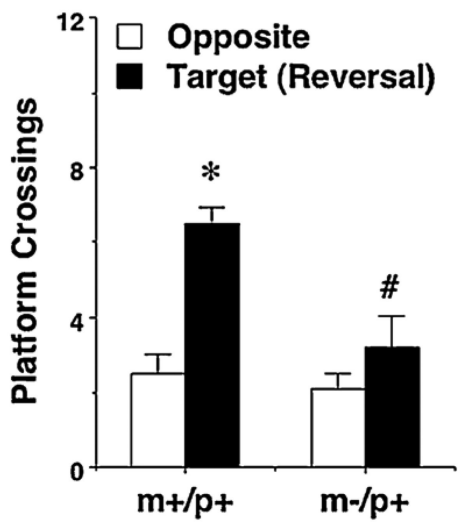

D) C57BL/6J (Cohort 1, 9 Weeks)

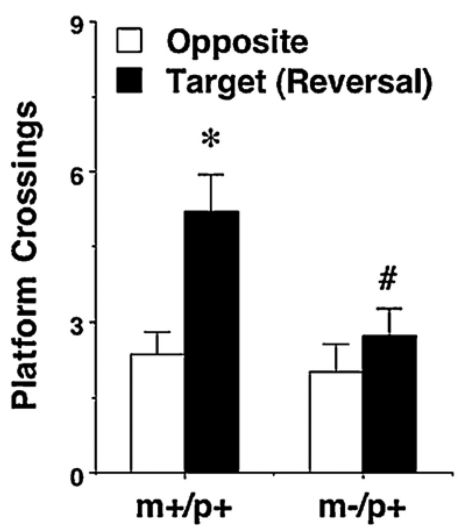

Fig. 8.

Selective target preference in the Morris water maze following acquisition and reversal. Each mouse was given a 1-min probe trial with the escape platform removed. Target (black bars) indicates the quadrant location where the platform was located during training trials. ${ }^{*} p<0.05$, within-group repeated measures ANOVA, significant main effect of quadrant. ${ }^{\#} p$ $<0.05$, post hoc comparison to $U b e 3 a^{m+/ p+}$, following repeated measures ANOVA across target and opposite quadrants, significant effect of genotype. 


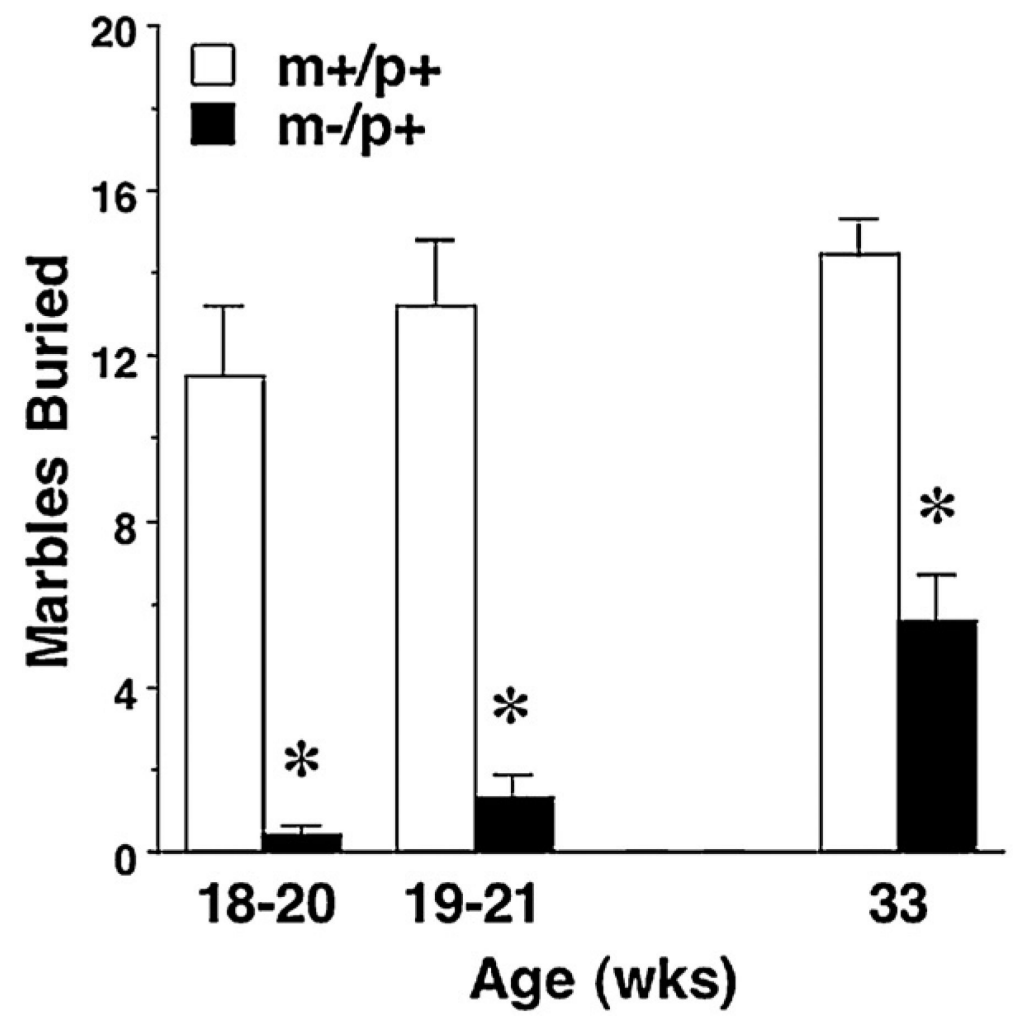

Fig. 9.

Reduced number of marbles buried by $U b e 3 a^{m-/ p+}$ mice in a digging task. Mice were from two separate cohorts on a C57BL/6J background. One cohort was given two tests, one week apart, at ages 18-20 and 19-21 weeks. The second cohort was given a single test at 33 weeks of age. For each test, mice were presented with 20 marbles for 30 -min. ${ }^{*} p<0.05$. 
A) Contextual Learning

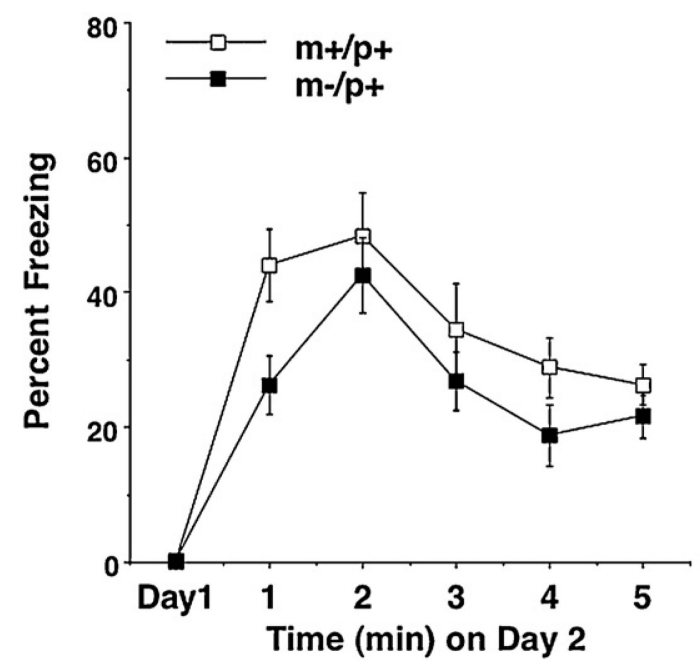

B) Cue Learning

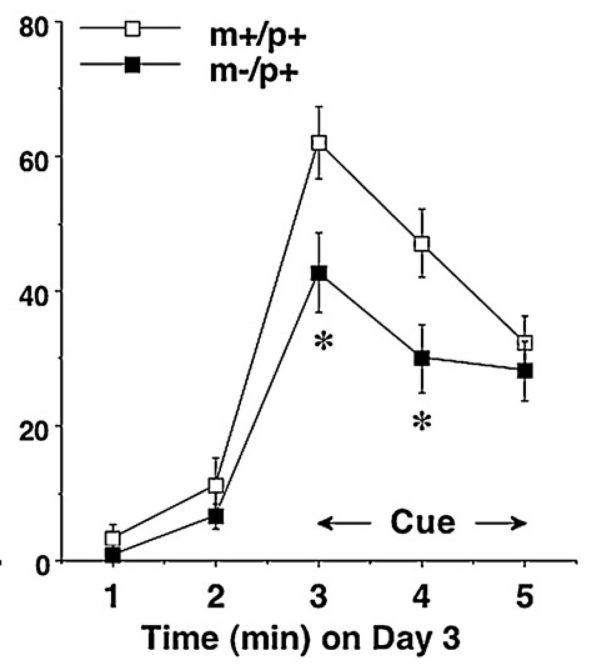

Fig. 10.

Impaired fear conditioning to an acoustic cue in $U b e 3 a^{m-/ p+}$ mice. Baseline levels of freezing behavior before shock exposure were determined on Day1 (the training day). Contextual learning was evaluated across 5 min on Day 2 of testing. Cue learning was determined on Day 3, using a 90 decibel acoustic stimulus. Subjects were on a C57BL/6J background, tested at $11-13$ weeks of age. Data shown are means $( \pm \mathrm{SEM}) . * p<0.05$. 
Table 1

Behavioral test regimens. Mice were on a 129S7/SvEvBrd-Hprt ${ }^{b-m 2}(129 / \mathrm{SvEv})$ or a C57BL/6J (B6) background. Assays included wire hang test for grip strength (Grip), activity (Act), rotarod (Rota), and prepulse inhibition of acoustic startle responses (PPI). Additional B6 cohorts were tested for marble burying (cohort 3; 18-20 and 19-21 weeks) and conditioned fear (cohort 4; 11-13 weeks).

\begin{tabular}{|c|c|c|c|c|}
\hline \multirow[t]{2}{*}{ Age (week) } & \multicolumn{4}{|c|}{ Background strain for $U b e 3 a$ maternal deletion } \\
\hline & 129/SvEv & B6, Cohort 1 & B6, Cohort 2 & B6, Cohorts 3 and 4 \\
\hline 5 & & Grip, Act, Rota & & \\
\hline 6 & & Sociability, PPI & & \\
\hline 7 & Grip & Grip, Act, Rota & & \\
\hline 8 & Act, Rota & Water Maze & & \\
\hline 9 & & Water Maze & & \\
\hline 10 & Sociability & Water Maze & & \\
\hline 11 & PPI & & & Conditioned Fear \\
\hline 13 & & & Rota & (Cohort 4) \\
\hline 14 & & Act, Rota & PPI & \\
\hline 15 & & & Act & \\
\hline 16 & & & Water Maze & \\
\hline 17 & & & Water Maze & \\
\hline 18 & & & & Marble Bury \\
\hline 19 & Water Maze & Rota & & Marble Bury \\
\hline 20 & Water Maze & & & (Cohort 3) \\
\hline 21 & Water Maze & & & \\
\hline 30 & & & Grip, Act & \\
\hline 31 & & & Rota & \\
\hline 33 & & & PPI, Marble Bury & \\
\hline
\end{tabular}




\section{Table 2}

Latency to a visible escape platform and swimming speed during the visual cue test in the Morris water maze. Data are means $( \pm \mathrm{SEM})$ of 4 trials per day.

\begin{tabular}{|c|c|c|c|c|}
\hline \multirow[t]{2}{*}{ Background strain } & \multicolumn{2}{|c|}{ Latency to platform (s) } & \multicolumn{2}{|c|}{$\underline{\text { Swim speed }(\mathrm{cm} / \mathrm{s})}$} \\
\hline & Day 1 & Day 2 & Day 1 & Day 2 \\
\hline \multicolumn{5}{|l|}{$129 / \mathrm{SvEv}^{a}$} \\
\hline $\mathrm{m}+/ \mathrm{p}+$ & $15.1 \pm 1$ & $11.0 \pm 2$ & $13.2 \pm 1$ & $15.0 \pm 1$ \\
\hline $\mathrm{m}-/ \mathrm{p}+$ & $23.7 \pm 3^{*}$ & $11.1 \pm 2$ & $13.3 \pm 1$ & $12.5 \pm 1^{*}$ \\
\hline \multicolumn{5}{|l|}{ C57BL/6J } \\
\hline \multicolumn{5}{|l|}{ Cohort 1 ; age 8 weeks } \\
\hline $\mathrm{m}+/ \mathrm{p}+$ & $21 \pm 3$ & $9 \pm 1$ & $18.9 \pm 0.8$ & $18.8 \pm 0.7$ \\
\hline $\mathrm{m}-/ \mathrm{p}+$ & $18 \pm 2$ & $11 \pm 2$ & $14.9 \pm 0.7^{*}$ & $15.1 \pm 0.9^{*}$ \\
\hline \multicolumn{5}{|l|}{ C57BL/6J } \\
\hline \multicolumn{5}{|c|}{ Cohort 2; age 16 weeks } \\
\hline $\mathrm{m}+/ \mathrm{p}+$ & $24.3 \pm 3$ & $18.0 \pm 2$ & $15.9 \pm 0.4$ & $16.1 \pm 0.6$ \\
\hline $\mathrm{m}-/ \mathrm{p}+$ & $25.4 \pm 2$ & $15.0 \pm 2$ & $12.0 \pm 0.4^{*}$ & $15.4 \pm 0.5$ \\
\hline
\end{tabular}

${ }^{a} 129 / \mathrm{SvEv}$ mice were approximately 20 weeks in age.

* $p<0.05$, comparison with $U b e 3 a^{m+/ p+}$ group. 